\title{
Coregistration of Interferometric Stacks of Sentinel-1 TOPS Data
}

\author{
Nestor Yague-Martinez, Francesco De Zan and Pau Prats-Iraola, Senior Member, IEEE
}

\begin{abstract}
The coregistration of SAR images is of fundamental importance for the generation of interferograms. The high azimuth coregistration requirements imposed by the TOPS acquisition mode imply that an advanced approach for the coregistration of stacked time series images is needed due to temporal decorrelation effects. In some scenarios, the conventional approach of estimating the shifts pairwise with respect to the same master might result insufficient. Therefore, a joint estimation is proposed here, which exploits jointly all interferograms in order to retrieve more accurate results. Simulated data and Sentinel-1A images acquired in IW (Interferometric Wide) mode are used to validate this procedure, demonstrating the better performance of the joint approach when compared to the standard single-master approach.
\end{abstract}

Index Terms-coregistration, Sentinel-1, TOPS, InSAR.

\section{INTRODUCTION}

$\mathbf{T}$ HE European Sentinel-1A (S1A) and Sentinel-1B (S1B) satellites were launched in April 2014 and April 2016, respectively. This constellation of SAR satellites was conceived to provide repeat-pass interferometric capabilities with unprecedented wide area coverage for medium resolution applications [1]. The SAR instrument, operating at $5.405 \mathrm{GHz}$, employs the Interferometric Wide (IW) swath mode for the systematic monitoring of large land and coastal areas. The IW mode is implemented as a three subswath TOPS (Terrain Observation by Progressive Scans) [2] SAR mode providing large swath widths of $250 \mathrm{~km}$ with a spatial resolution of $5 \mathrm{~m}$ x $20 \mathrm{~m}$ in the range and azimuth dimensions, respectively.

The necessary high azimuth coregistration requirements for TOPS data due to the nature of the burst-mode acquisition and the azimuth antenna steering have already been pointed out [3][4]. In [5] details on the interferometric processing of Sentinel-1 TOPS SAR images pairs are provided indicating that a coregistration accuracy of approximately 0.0009 samples (equivalent to ca. $1.9 \mu \mathrm{s}$ or $1.3 \mathrm{~cm}$ ) is required to limit the InSAR phase error to $1 / 100$ cycle. The established method to achieve the necessary azimuth coregistration accuracy is based on Enhanced Spectral Diversity (ESD) [4], which exploits the spectral separation of the data in the overlapping areas between adjacent bursts.

When applying advanced techniques for displacement measurement, such, e.g., Persistent Scatterers Interferometry (PSI), it is necessary to have the images of the stack coregistered to a common master image. The state-of-the-art procedure consists

The authors are with the German Aerospace Center (DLR), Oberpfaffenhofen, D-82234, Germany. (e-mail: nestor.yague@dlr.de)

This work was supported in part by the European Space Agency's SEOM Programme under Contract 4000110587/14/I-BG in performing first a geometrical coregistration between all slaves and the master image using precise orbit information and an external Digital Elevation Model. Afterwards baseline errors or orbital/instrument timing errors can be determined by exploiting the SAR data.

In this paper we will focus on the determination of the (rigid) along-track shifts for S1 TOPS images stacks. The coregistration problem consists in retrieving the azimuth shift of each image with respect to a common master image. The single-master approach applies ESD between each image and the master image. Since the ESD performance depends on the coherence, it is expected that the performance of the estimation drops with the temporal baseline. A joint coregistration approach appears to be the optimal solution when working with long stacks. This approach has been already addressed in the literature for the case of coherently correlating speckle signals [6]. The same principle can be applied when employing the ESD technique for azimuth coregistration of TOPS images [7][8][9]. This paper is an extended and updated version of the work presented in [8]. A similar procedure employing subsets of the total number of images of the stack has been proposed recently in [10]. Other interesting option is to employ only Point Scatterers for the ESD estimation, which keep a high coherence over time. The performance of the estimation would however depend on the density of scatterers that are imaged in the overlap areas. Moreover since the Sentinel-1 mission was devised to map wide areas, where for some cases no urban regions are present, a more general solution is required.

The paper is structured as follows: Section II provides the methodology to perform a joint coregistration by exploiting ESD together with a Weighted Least Squares (WLS) approach. Section III evaluates the performance of this estimator and compares it to the Phase Linking (PL) algorithm by employing Monte-Carlo simulations. In section IV results with a stack of S1A data over Mexico are provided; in Section IV-A the performance of the joint coregistration approach over the single master approach in terms of retrieved shifts variance is exposed; Section IV-B makes a quantitative comparison of the shifts obtained between the direct and joint approaches. In section IV-C a validation is performed exploiting the long-term coherence properties at urban areas. After having validated the method with S1A data not presenting ground displacements in the North-South direction, in Section V a suitable solution for scenarios presenting ground displacements is briefly discussed. The conclusions are drawn in Section VI. 


\section{Methodology}

In section II-A the ESD estimator is introduced. Section II-B introduces the joint coregistration approach based on WLS.

\section{A. Enhanced Spectral Diversity}

ESD [4] exploits the phase difference in the overlap area between adjacent bursts. The ESD phase can be calculated for every sample, $p$, of each overlap area as follows:

$$
\phi_{\mathrm{ESD}, \mathrm{p}}=\arg \left\{\left(m_{i} \cdot s_{i}^{*}\right) \cdot\left(m_{i+1} \cdot s_{i+1}^{*}\right)^{*}\right\},
$$

where $m_{i}$, and $s_{i}$ refer to the $i^{t h}$ master and slave complex bursts, and $m_{i+1}$, and $s_{i+1}$ refer to the $(i+1)^{t h}$ master and slave bursts; $\arg \{\cdot\}$ gives the phase of a complex number.

The azimuth shift can be retrieved by exploting following periodogram, equivalently as in [5]:

$$
\widehat{\Delta y}=\underset{\Delta y}{\arg \max }\left\{\Re\left(\sum_{p} e^{j\left(\phi_{\mathrm{ESD}, \mathrm{p}}-2 \pi \Delta f_{\mathrm{DC}, \mathrm{p}}^{\mathrm{ovl}} \frac{\Delta y}{f_{\mathrm{az}}}\right)}\right)\right\},
$$

where $\arg \max _{\Delta y}\{\cdot\}$ stands for the argument of the maximum (azimuth shift, $\Delta y$, for which the function attains its largest value). $\Delta f_{\mathrm{DC}, \mathrm{p}}^{\mathrm{ovl}}$ is the Doppler centroid frequency difference in the overlap areas for each sample, $p$, and $f_{\text {az }}$ is the image azimuth sampling frequency.

\section{B. Joint coregistration}

Among the different possibilities to jointly estimate the azimuth shifts we can differentiate between methods working with the ESD interferograms (complex domain) or the derived shifts (real numbers). More precisely, the Phase Linking (PL) algorithm [11] provides a joint estimation of the phases whereas a Weighted Least Squares (WLS) procedure works with retrieved shifts. Phase Linking is the maximum likelihood estimator of the phases in a stack, which exploits all possible interferogram combinations ( $N^{2}$, having $N$ acquisitions). PL requires the coherence matrix, which is estimated from the data.

In our case shifts of only a few centimeters are expected, mainly coming from inaccuracies of the orbit product. Thus, having into account that the ESD ambiguity band is about $\pm 71 \mathrm{~cm}$ in the worst case [5], we could assume that the shifts from each pair are not affected by wrapping effects. The requirement for this implies that a certain coherence is available, which should be the case when working with real data. Moreover a sufficient number of samples have to be averaged in order to avoid wrapping effects due to phase noise. This is also fulfilled since we can employ all samples of all overlapping areas of the scene. This enables the use of WLS to jointly estimate the shifts of the stack. In section $\mathrm{V}$ we propose the use of PL for an scenario with ground deformation in the sensor along-track direction.

In the first place, all images are coarsely coregistered to a common master using geometric information. In order to achieve this, the use of precise (PREC) or restituted (RES) orbits is recommended since they provide an accuracy in the order of a few centimeters in the along-track dimension [5], which is within the main ambiguity band of ESD. Afterwards

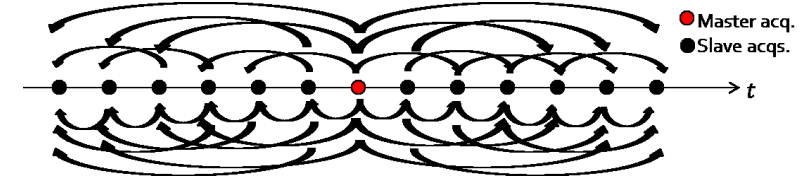

Fig. 1. In the joint coregistration procedure the mutual shifts among all available pairs are calculated. This way the temporal decorrelation effect can be mitigated. In the standard single-master approach uniquely the shifts between each slave and a selected master, usually chosen in the middle of the stack, is performed. The joint approach supposes moreover additional flexibilities regarding the selection of the master image.

mutual ESD estimation can be applied among all possible pairs, as shown in Figure 1. All samples of the overlapping areas are used in the ESD step, providing a robust estimation against phase wrapping effects (in the case of phase noise due to low coherence). Note that an outlier rejection procedure, e.g., based on the coherence, can be applied to avoid averaging pure noise samples.

The azimuth shifts, $\mathbf{m}_{\mathrm{L}_{2}}$, of each image with respect to the master image can be calculated using:

$$
\mathbf{m}_{\mathrm{L}_{2}}=\left(\mathbf{G}^{T} \mathbf{W G}\right)^{-1} \mathbf{G}^{T} \mathbf{W d}
$$

where $\mathbf{G}$ is the system matrix defining the relations between the different measurements, $\mathbf{d}$ the data vector (pairwise ESD measurements). $\mathbf{W}$ is a diagonal matrix including the weights (inverse of the shift variance of the pairwise measurements, that can be computed from the estimated coherences). In [8] a detailed description of the system of equations can be found for the case where the master image is the first of the stack. A possibility to limit the computational burden when working with long time series is to exploit subsets of images of the stack, e.g., by setting a maximum time span. However, this strategy would not profit from distant high coherent images in the presence of seasonal effects or long-term coherence, and represents a sub-optimal solution in terms of performance.

The Weighted Least Squares method has been described so far. However, being rigorous, the correlation between shift estimates should be considered as well. The covariance between shifts (in seconds) can be computed as [12]:

$$
E\left\{d_{n, k} d_{h, l}\right\}=\frac{\gamma_{\mathrm{nh}} \gamma_{\mathrm{kl}}-\gamma_{\mathrm{nl}} \gamma_{\mathrm{kh}}}{L \gamma_{\mathrm{nk}} \gamma_{\mathrm{hl}}\left(2 \pi \Delta f_{\mathrm{DC}}^{\mathrm{ov}}\right)^{2}}
$$

where $L$ is the effective number of looks of the estimation window and $\gamma_{\mathrm{ij}}$, is the interferometric coherence between image $i$ and image $j$.

The weight matrix, $\mathbf{W}$, should then be filled with the corresponding covariance values and would become a variancecovariance matrix. The inversion problem employing the variance-covariance matrix becomes a Generalized Least Squares (GLS) one. A feasible GLS (FGLS) solution can be obtained using the Prais-Winsten or Cochrane-Orcutt estimator [13]. This is however out of the scope in this letter.

It is interesting to note that if the stack is large enough, the mean of all estimated shifts corresponds to the along-track geolocation error of the master image, which occurs mainly due to the error in the master orbit. Therefore, in such cases one can also use this joint-estimator to evaluate the quality of the orbit products (at least in the along-track direction). 


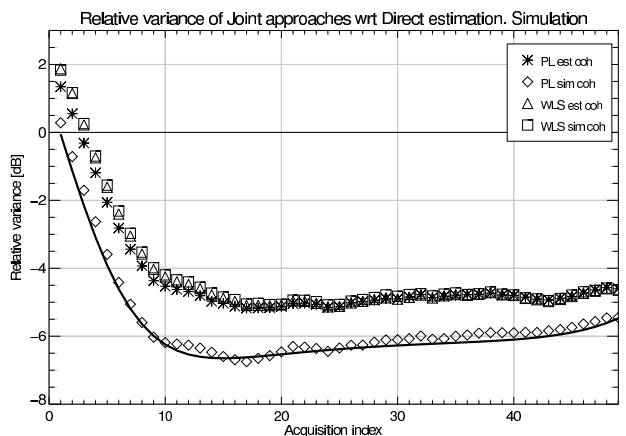

Fig. 2. Relative variance of Phase Linking and Weighted Least Squares estimators with respect to the direct estimation employing simulated data. A Monte-Carlo simulation was done assuming an exponential decorrelation model with a constant time of 40 days and a long-term coherence of 0.2 for a stack of 50 images acquired consecutively with a repetition cycle of 12 days. The master image is chosen at the beginning of the time-series (index 0 ). The solid red line corresponds to the relative variance of the Cramér-Rao bound of the joint estimator over the direct estimator. The relative variance decreases linearly with the number of images until a certain number where it almost saturates. Negative values of the plot mean a reduction of the standard deviation of the joint approaches in comparison to the single-master estimator. The actual point in which the behavior changes is related to the particular relationship between the decorrelation constant time and the repetition cycle of the system.

\section{JOINT COREGISTRATION APPROACHES AND Achievable Performance With Simulated Data}

In order to evaluate the performance of the WLS estimator a Monte-Carlo simulation for a stack of 50 images has been carried out. The master image corresponds to the first acquisition. For the simulation, an exponential decorrelation model [14] with a time constant of 40 days and a longterm coherence of 0.2 was assumed. Fig. 2 shows the gain of the joint approach employing WLS and Phase Linking with respect to the direct estimation. The solid red line corresponds to the Cramér-Rao bound [11]. Note that the estimation result employing two sources of coherences for the covariance matrix have been used: the one estimated from the simulated data (est coh) and the theoretical one used to generate the simulated data (sim coh). We can see that in the case of using Phase Linking, the curve approaches the bound if the theoretical coherence is used. If the estimated coherence is used, there is a gain loss. In the case of WLS, we cannot appreciate significant differences employing one or the other and, moreover, the performance is very similar to the one achieved by PL with the estimated coherence. We conclude that employing WLS represents a good solution for the retrieval of the azimuth shifts.

\section{Application to S1 DATA}

We have selected a data set of a total of 43 S1A IW acquisitions over Mexico for the evaluation of the procedure. The City of Mexico is covered by one of the subswaths, whereas one of the remaining subswaths corresponds mainly to non-urban areas. This makes the scenario suitable to perform a validation of the method since long-term coherent as well as rapidly decorrelating scatterers can be found. The images were acquired between November 2014 and August 2016 in ascending geometry (track 78). The master image, acquired on

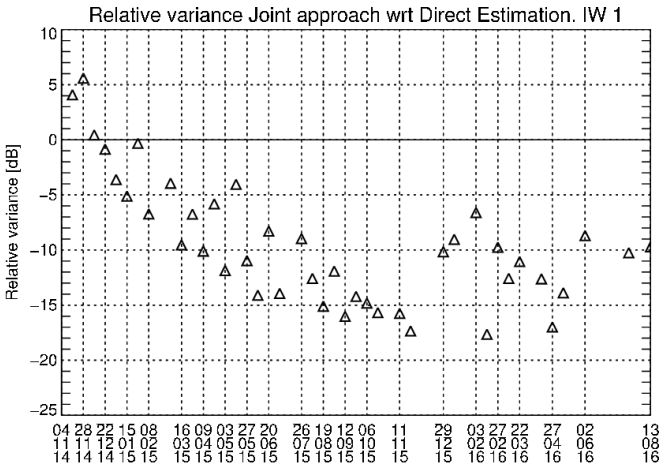

Fig. 3. Relative variance, in $\mathrm{dB}$, of the joint estimator over the single-master estimator for the IW1 subswath over Mexico site (distributed scatterers). Negative values of the plot mean a reduction of the standard deviation of the joint estimator in comparison to the single-master estimator. The gain of the joint estimation raises as the temporal baseline increases.

November 4, 2014, has been chosen at the beginning of the stack in order to maximize temporal decorrelation effects.

\section{A. Joint Estimator Performance Evaluation}

The first assessment that we present corresponds to the comparison of the standard deviation of the estimated shifts with the single-master and joint approaches. We focus on the IW1 subswath, which covers non-urban areas. Each overlap area of the subswath is divided into blocks and the joint estimator is applied to each block. The performance of the estimation is calculated as the quotient between the standard deviation of the joint estimation and the one of the singlemaster approach. Fig. 3 shows the relative variance, in $\mathrm{dB}$, of the joint estimator over the single-master estimator for areas affected by temporal decorrelation. Negative values of the plot mean a reduction of the standard deviation of the joint estimator in comparison to the single-master estimator. It can be observed that the gain of the joint approach raises as the temporal baseline increases for this kind of scatterers.

\section{B. Comparison between Joint Coregistration and single- master approaches}

A quantitative comparison between the shifts obtained with joint and single-master approaches is shown in the following. We focus now on the IW3 subswath, which covers urban and non-urban areas. Figure 4 shows the coherence at the overlapping areas of this subswath for the largest temporal baseline of the stack (504 days). It can be appreciated that the third overlap contains urban (City of Mexico) and non-urban areas making it convenient for a validation of the joint method in terms of retrieved shift values. Figures 5 and 6 show the exponential fitting that has been performed together with the covariance matrices for exemplary targets located in urban area and over land, respectively. The decorrelation model proposed in [14] has been used, which provides three parameters: initial coherence, i.e. coherence for 0 days lag, time constant and long-term coherence.

It can be seen that the targets over urban areas present longterm coherence whereas distributed scatterers have a faster decay of the coherence with time. Taking advantage of this 


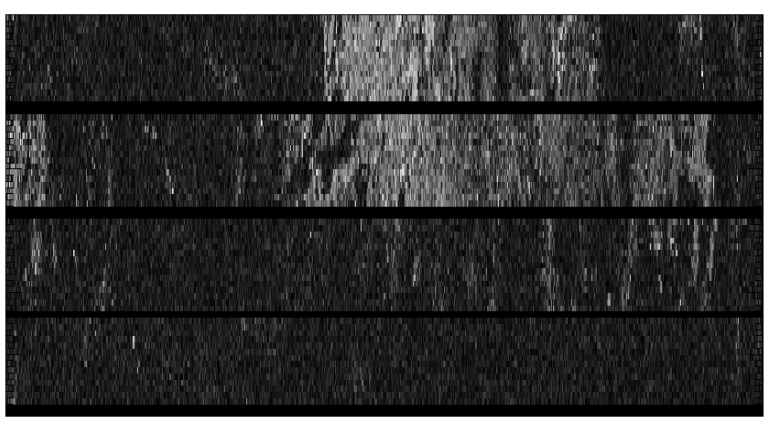

Fig. 4. Coherence of the forward look at the overlap areas of IW3 for the pair 04/11/2014-13/08/2016, corresponding to a temporal baseline of 504 days. We focus on the third overlap area, which cover the City of Mexico, where longterm coherence properties can be appreciated.
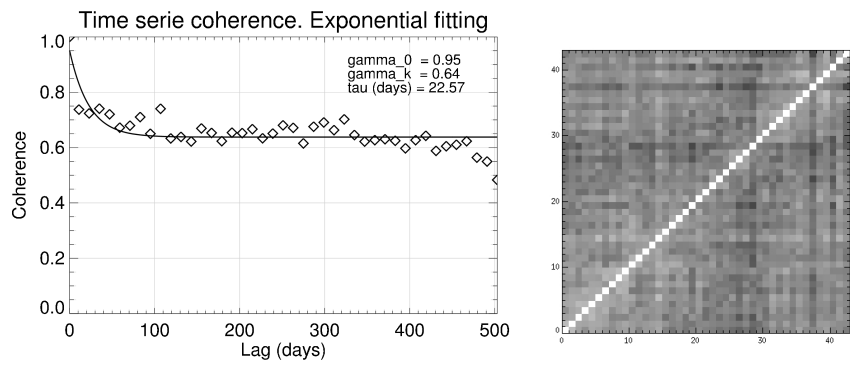

Fig. 5. Modeling of the temporal decorrelation for an urban target
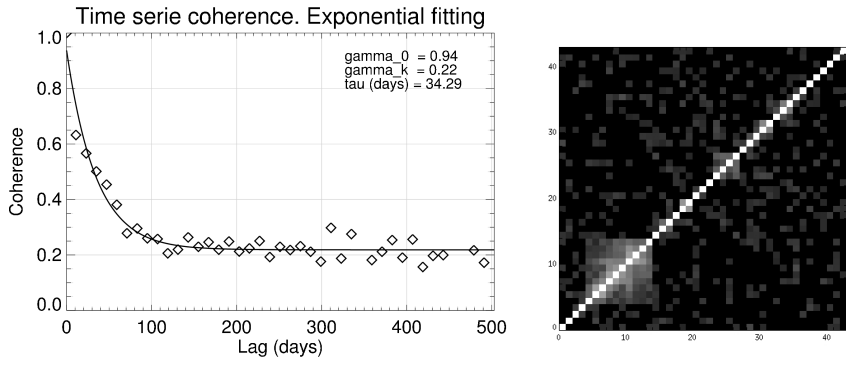

Fig. 6. Modeling of the temporal decorrelation for a target over land area

fact we generate a mask for the urban areas (establishing a threshold to the coherence between the master image and the slave image with the largest temporal span) and estimate the shifts using the joint and single-master approach. Fig. 7 shows the difference between direct ESD and the joint retrieved shifts for the urban area. The deviation of the direct ESD shifts with respect to the joint solution is very small. This is an expected result since urban areas present long-term coherence properties and the single-master estimate already provides good results.

If we mask out the urban areas and consider only the areas with distributed scatterers we obtain the plot of Fig. 8 showing much larger differences between direct ESD and the joint retrieved shifts over the land areas. We assume that the joint estimates are the reference since they correspond to the optimal estimation. The required 0.0009 samples values have been depicted with blue dashed lines and it can be observed that these limits are exceeded for some of the acquisitions, being especially large when the temporal baseline increases. It should be expected to have interferometric phase errors of about 10 degree for some acquisitions, e.g., on 10/03/2016 or

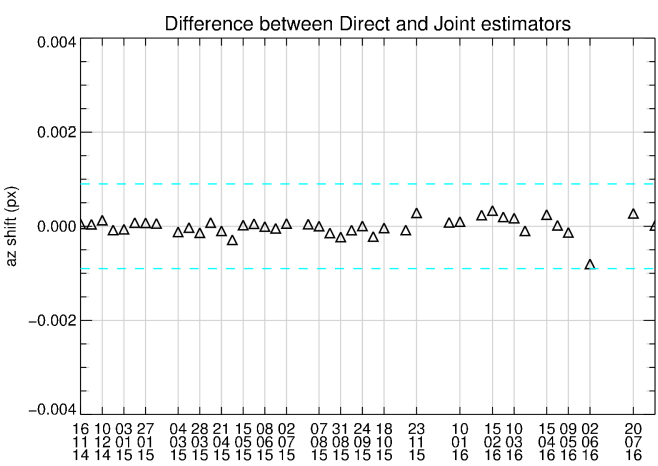

Fig. 7. Comparison between the azimuth shifts retrieved with the joint coregistration approach and the single master approach over urban areas. The blue dashed lines correspond to the required 0.0009 samples accuracy.

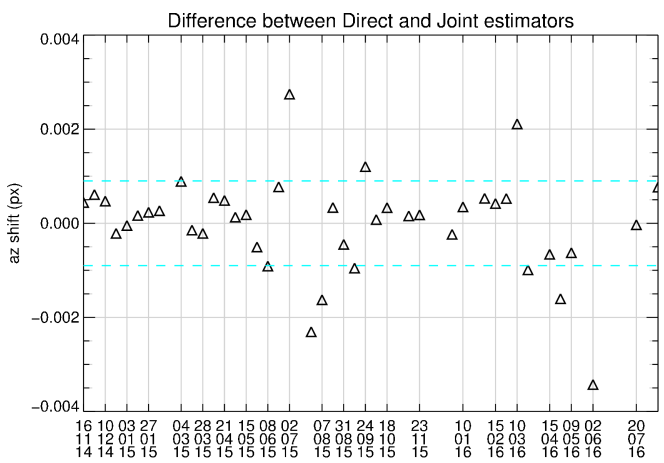

Fig. 8. Comparison between the azimuth shifts retrieved with the joint coregistration approach and the single master approach over distributed targets (non-urban areas). The blue dashed lines correspond to the required 0.0009 samples accuracy.

02/06/2016. These two acquisitions will be further analysed in the next section.

We have compared the shift for two scenarios: (i) urban area, and (ii) non-urban area. The gain of the joint estimation is evident for non-urban areas. In the case of sites which present long-term coherence properties, it is also a matter of "luck" if these regions are mapped by the burst overlap areas. The estimation of the decorrelation parameters, especifically the long-term coherence agree with the obtained results.

\section{Validation - Interferogram of a selected pair}

We can make more evident the phase error that we would have in case that we generate the interferogram of a selected pair, e.g. in a SBAS scenario, for the different coregistration approaches. From Fig. 8 we can see that the acquisitions on 10/03/2016 and 02/06/2016 present large deviations between both estimates. We evaluate in the following the residual ESD phase - measured in a direct way - over the urban area. This area presents long-term coherence, as shown in Fig. 5, being the direct estimation reliable. We perform this measurement for three different coregistered versions of the images: (i) only geometric coregistration, (ii) single-master approach and (iii) joint approach. For (ii) and (iii) the non-urban area was used to retrieve the azimuth shifts with respect to the master image. The results are summarised in Fig 9. The figure shows the histograms of the measured ESD phases for all three cases. The mean value of the ESD phase in case that only a geometric 


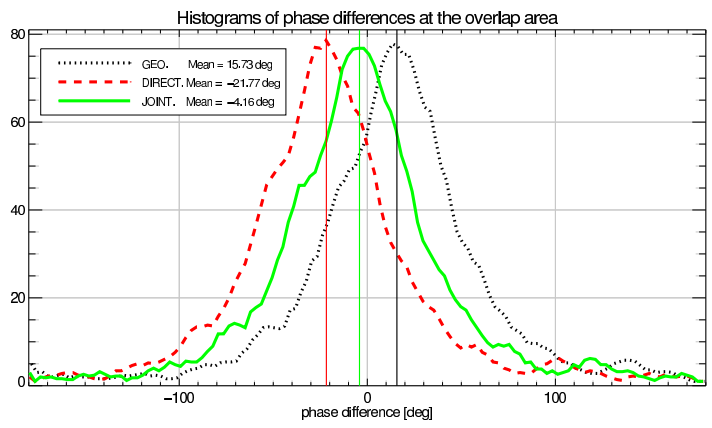

Fig. 9. Histograms of the measured residual ESD phases for the three coregistration possibilities. The histogram of the residual ESD phase in case that only a geometric coregistration is performed is shown with a black dotted line, presenting a mean value of $15.73^{\circ}$. The histogram in case that the azimuth shifts are calculated employing the single-master approach is shown with a red dashed line, providing a residual ESD phase equal to $-21.77^{\circ}$ The histogram of the residual ESD phase for the images coregistered jointly is shown with a green solid line. In this case the mean error is only $-4.16^{\circ}$ which is much closer to our established boundaries

coregistration is performed is $15.73^{\circ}$, larger than the targeted $3.6^{\circ}$. This phase error is due to timing/orbital errors of both acquisitions. In case that the azimuth shifts are calculated employing the single-master approach an "overcorrection" takes place, delivering a residual ESD phase equal to $-21.77^{\circ}$, which is even a larger error than the one corresponding to the orbital error. The joint estimate delivers a residual ESD phase of only $-4.16^{\circ}$ which is much closer to our established boundaries.

\section{DISCUSSION}

We have assumed so far that in the scene no local displacement in the along-track direction occurs. However this is, in general, not realistic, since time-series analyses are usually intended to monitor displacements. As the temporal baseline increases, these displacements can become important and cannot just be attributed to orbital errors. Our proposal to cope with this situation performing simultaneously a joint coregistration is now briefly described. A PLinking procedure can be applied to the ESD phases at the overlapping areas in order to retrieve the maximum likelihood estimate of the phases. PL with a small multilook window is used in order to be adaptive to local displacements, since note that the WLS approach averages all valid samples of all overlap areas. For each multilooked pixel, the ESD phases would contain two contributions: one due to the orbital error and one due to the ground displacement. The periodogram can be applied to obtain the mean azimuth deformation rate. Once the slope is retrieved, the values can be de-trended in order to isolate the orbital errors. An average of the "de-trended" values employing all multilooked samples for each pair would provide the corresponding azimuth shift due to an orbital error. Note that the result also corresponds to a joint estimate, since we applied Phase Linking to the ESD phases.

\section{CONCLUSIONS}

In this work we addressed the azimuth coregistration problem of interferometric stacks of TOPS images using S1 IW data. The high coregistration requirements for TOPS data have been stressed, highlighting the necessity of sophisticated methods when coregistering long stacks of images. The joint coregistration idea has been applied to the retrieval of the (rigid) azimuth shifts applying a least squares estimate. Results with Monte-Carlo simulations show that for the retrieval of the rigid azimuth shifts the least squares estimate provides very similar performance to Phase Linking.

The better performance of the joint estimation has been demonstrated with a stack of 43 S1A images over Mexico. The best results are achieved when mapping land areas, where temporal decorrelation effects are important. A comparison of the joint estimation with the single-master approach has been done indicating that deviations of more than 0.0009 samples can occur if a joint approach is not used. These errors introduce biases in the interferometric phases, as shown for the analysed stack, increasing the noise of the line-of-sight deformation measurements over time. The higher the density of persistent scatterers at the overlap areas is, the lower is the gain of the join estimator. However applying a joint estimation procedure provides, in general, a better estimation of the shifts.

\section{REFERENCES}

[1] R. Torres, P. Snoeij, D. Geudtner et al., "GMES Sentinel-1 mission," Remote Sensing of Environment, vol. 120, no. 0, pp. 9 - 24, 2012, the Sentinel Missions - New Opportunities for Science. [Online]. Available: http://www.sciencedirect.com/science/article/pii/S0034425712000600

[2] F. De Zan and A. Monti Guarnieri, "TOPSAR: Terrain Observation by Progressive Scans," Geoscience and Remote Sensing, IEEE Transactions on, vol. 44, no. 9, pp. 2352-2360, Sept 2006.

[3] R. Scheiber and A. Moreira, "Coregistration of interferometric SAR images using spectral diversity," Geoscience and Remote Sensing, IEEE Transactions on, vol. 38, no. 5, pp. 2179-2191, Sep 2000.

[4] P. Prats-Iraola, R. Scheiber, L. Marotti et al., "TOPS Interferometry With TerraSAR-X," Geoscience and Remote Sensing, IEEE Transactions on, vol. 50, no. 8, pp. 3179-3188, Aug 2012.

[5] N. Yague-Martinez, P. Prats-Iraola, F. R. Gonzalez et al., "Interferometric Processing of Sentinel-1 TOPS Data," IEEE Transactions on Geoscience and Remote Sensing, vol. 54, no. 4, pp. 2220-2234, April 2016.

[6] F. De Zan, "Coherent Shift Estimation for Stacks of SAR Images," Geoscience and Remote Sensing Letters, IEEE, vol. 8, no. 6, pp. 10951099, Nov 2011.

[7] F. De Zan and P. Prats-Iraola, "Joint Coregistration of SAR Images with an Application to TerraSAR-X TOPS Mode Datasets," in Living Planet Symposium 2013, Edinburgh, UK.

[8] N. Yague-Martinez, P. Prats-Iraola, and F. De Zan, "Coregistration of Interferometric Stacks of Sentinel-1A TOPS Data," in Proceedings of EUSAR 2016: 11th European Conference on Synthetic Aperture Radar, June 2016, pp. 1-6.

[9] M. Nannini, P. Prats-Iraola, F. D. Zan et al., "TOPS Time Series Performance Assessment With TerraSAR-X Data," IEEE Journal of Selected Topics in Applied Earth Observations and Remote Sensing, vol. 9, no. 8, pp. 3832-3848, Aug 2016.

[10] H. Fattahi, P. Agram, and M. Simons, "A Network-Based Enhanced Spectral Diversity Approach for TOPS Time-Series Analysis," IEEE Transactions on Geoscience and Remote Sensing, vol. 55, no. 2, pp. 777-786, Feb 2017.

[11] A. Monti Guarnieri and S. Tebaldini, "On the Exploitation of Target Statistics for SAR Interferometry Applications," Geoscience and Remote Sensing, IEEE Transactions on, vol. 46, no. 11, pp. 3436-3443, Nov 2008.

[12] F. D. Zan, M. Zonno, and P. Lopez-Dekker, "Phase Inconsistencies and Multiple Scattering in SAR Interferometry," IEEE Transactions on Geoscience and Remote Sensing, vol. 53, no. 12, pp. 6608-6616, Dec 2015.

[13] C.-M. Kuan, "Generalized Least Squares Theory," 2004.

[14] F. Rocca, "Modeling interferogram stacks," Geoscience and Remote Sensing, IEEE Transactions on, vol. 45, no. 10, pp. 3289-3299, Oct 2007. 